\title{
Hantavirus en roedores de la Octava Región de Chile
}

\author{
Hantavirus in rodents of the VIII Region of Chile \\ JUAN CARLOS ORTIZ1, WALDO VENEGAS ${ }^{2}$, JOHN A. SANDOVAL ${ }^{3}$, \\ PATRICIO CHANDÍA ${ }^{3}$ \& FERNANDO TORRES-PÉREZ ${ }^{1,4}$ \\ ${ }^{1}$ Departamento de Zoología, Universidad de Concepción, Casilla 160-C, Concepción, Chile; e-mail: jortiz@udec.cl \\ ${ }^{2}$ Facultad de Ciencias Biológicas, Universidad de Concepción, Chile \\ ${ }^{3}$ Departamento de Ciencias, Universidad del Biobío, Chillán, Chile \\ ${ }^{4}$ Departamento de Ecología, Facultad de Ciencias Biológicas, Pontificia Universidad Católica de Chile, \\ Casilla 114-D, Santiago, Chile
}

\begin{abstract}
RESUMEN
La Octava Región de Chile corresponde a la segunda región con el mayor número de casos de Síndrome Cardiopulmonar por Hantavirus (SCPH). Por tal motivo se realizó un estudio para detectar la presencia de anticuerpos contra hantavirus en roedores y su distribución local en la Octava Región. El estudio comprendió las cuatro provincias de la región y consideró once sitios de muestreo. Se capturaron siete especies de roedores, Abrothrix olivaceus fue la más abundante seguida de Oligoryzomys longicaudatus. De los 300 roedores analizados, cinco ejemplares $(1,66 \%)$ resultaron ser positivos a hantavirus y correspondieron a tres especies de sigmodontinos: a saber, Loxodontomys micropus, que corresponde al único registro de este tipo para la especie en Chile, Abrothrix longipilis y Oligoryzomys longicaudatus.
\end{abstract}

Palabras clave: Hantavirus, Oligoryzomys longicaudatus, VIII Región, Chile, serología.

\begin{abstract}
The Eight Region has the second highest number of cases of Hantavirus Cardiopulmonary Syndrome (HCPS) in humans for Chile. A study was performed to identify the number of rodents serologically positive to hantavirus and their local distribution in this region. To achieve this goal, we sampled eleven sites in the four provinces of the region. Seven rodents species were collected, with Abrothrix olivaceus presenting the largest number of captures followed by Oligoryzomys longicaudatus. Of the 300 rodents analyzed, five (1.66 \%) were sero-positives to hantavirus and belonged to three different sigmodontine species: Abrothrix longipilis, O. longicaudatus, and Loxodontomys micropus. No previous records of seropositive L. micropus existed.
\end{abstract}

Key words: Hantavirus, Oligoryzomys longicaudatus, Eight Region, Chile, serology.

\section{INTRODUCCIÓN}

El Síndrome Cardiopulmonar por Hantavirus $(\mathrm{SCPH})$ es considerado una de las enfermedades emergentes más peligrosas en América (Mills \& Chids 1998), conocida en el continente a partir de un brote en el suroeste de los Estados Unidos en 1993. El primer caso registrado en Chile se produjo en 1995 en Cochamó, Décima Región (Toro et al. 1998), aunque retrospectivamente se han determinado casos en 1993 y 1975 (Baró et al. 1999). Esta enfermedad es provocada por un virus del género Hantavirus (Bunyaviridae), el cual se encuentra hospedado en roedores de la familia Muridae. En América, los principales reservorios de este virus se encuentran dentro de la subfamilia Sigmodontinae. Además, se ha señalado que existe un alto grado de especificidad entre los reservorios (roedores) y sus respectivos hospederos (Schmaljohn \& Hjelle 1997), lo que sugiere que la enfermedad sería de larga data y que habría ocurrido coevolución entre las diversas cepas descritas y su reservorio (Yates et al. 2002).

En Chile, cinco especies de sigmodontinos han sido registradas seropositivas a hantavirus (Pavletic 2000, Spotorno et al. 2000). De estas, Oligoryzomys longicaudatus (Bennett 1832) ("ratón colilargo") es el principal reservorio de la cepa Andes (Levis et al. 1998, Toro et al. 1998). Este roedor se distribuye en Chile desde la Región de Atacama $\left(28^{\circ} \mathrm{S}\right)$ hasta Aysén $\left(50^{\circ} \mathrm{S}\right)$ y en los bosques patagónicos de Argentina (Gallardo \& Palma 1990, Gonzáles-Ittig et al. 2002). Los hábitats más utilizados para esta especie son los ambientes boscosos y de mato- 
rral, en lugares húmedos y de vegetación densa (Gonzáles et al. 2000). Además, las especies Abrothrix olivaceus (Waterhouse, 1837), Abrothrix longipilis (Waterhouse, 1837), Phyllotis darwini (Waterhouse, 1837) y Loxodontomys micropus (Waterhouse, 1837) (dato para esta última especie extraído a partir de este estudio) parecen presentar una tasa menor de seroprevalencia (E. Palma comunicación personal). Estas últimas especies entrarían en contacto con el virus desde el reservorio primario mediante "spill-over" (Hjelle \& Yates 2001) aunque se desconoce su grado de participación en la transmisión hacia los humanos.

Desde 1995 y hasta antes de este estudio (25 agosto 1998), se disponía de la confirmación de 68 casos de Síndrome Cardiopulmonar por Hantavirus en Chile (Toro et al. 1998). Sin embargo, el número de casos acumulados hasta diciembre de 2002 ha alcanzado a 277, 66 de estos en la Octava Región, lo que convierte a esta región en la segunda con mayor número de casos después de la Décima Región (http:// epi.minsal.cl).

Con el propósito de establecer la presencia de roedores positivos a la cepa Andes de Hantavirus y su distribución regional, se realizó un estudio de captura y determinación de las especies involucradas en las cuatro provincias de la Octava Región de Chile.

\section{MATERIALES Y MÉTODOS}

Se muestrearon 11 sitios de las cuatro provincias de la región (Arauco, Biobío, Concepción, Ñuble) entre marzo y junio de 1998 (Fig. 1, Tabla 1). Los sitios fueron seleccionados de acuerdo a los siguientes criterios: disponibilidad de ejecución del muestreo, ambientes mésicos cuyas características vegetacionales formarían parte del hábitat de O. longicaudatus (Gonzáles et al. 2000; Murúa et al. 1987), y en sitios con registros de roedores por parte de lugareños. Se enfatizaron sectores con vegetación nativa (e.g., Las Trancas), presencia de Chusquea quila (quila), cercanos a plantaciones de pino, eucaliptos o aromo, localidades donde previamente se han registrado contagios con Hantavirus, y áreas con presencia permanente o temporal de población humana (escuelas rurales, parques, zonas de recreación y parcelas de agrado; e.g., Contulmo, Parque Botánico Hualpén). Se utilizaron trampas Sherman de captura viva estándar ( $8 \times 9 \times 23 \mathrm{~cm})$, las que fueron puestas en situaciones peridomiciliarias, en bosques, praderas, plantaciones y a lo largo de cercas y caminos. Las trampas fueron operadas durante dos (alto éxito de captura) o cuatro noches (bajo éxito de captura). El esfuerzo de trampeo varió desde 160 a 1.400 trampas noche (Tabla 1). Para la captura y manipulación de los roedores se siguieron las normas de procesamiento de acuerdo a los protocolos del Centro de Enfermedades Infecciosas y Prevención de Atlanta (Mills et al. 1998). La disposición espacial de las trampas se realizó de acuerdo a transectos de 10 trampas distanciadas $5 \mathrm{~m}$ una de otra; los transectos se ubicaron a una distancia de 10 m entre sí. Se evaluó la densidad relativa de roedores de acuerdo al éxito de captura mediante el número de trampas/noche. Una vez capturados, los animales fueron anestesiados y se les extrajo una muestra de sangre desde el sinus retroorbital por medio de un capilar heparinizado. Las muestras de sangre fueron almacenadas en nitrógeno líquido y transportadas al laboratorio para su posterior análisis serológico. Los especímenes fueron preliminarmente determinados en el campo y confirmados en el laboratorio a nivel específico de acuerdo a las claves de Osgood (1943) y Mann (1978). Además, a cada ejemplar se le extrajo una muestra de riñón, bazo, hígado y pulmón, las cuales fueron almacenadas en nitrógeno líquido y fueron utilizadas para confirmar la presencia del virus. Los especímenes fueron fijados y luego preservados en alcohol $70 \%$, y finalmente almacenados en el Museo de Zoología de la Universidad de Concepción. Los análisis serológicos para Hantavirus se realizaron en el Instituto de Salud Pública de Chile (Sección Virología Clínica) mediante "positive enzyme-linked immunosorbent assay" IgG, ELISA (Elgh et al. 1997).

\section{RESULTADOS}

Se capturó un total de siete especies de roedores de la familia Muridae, cinco de las cuales correspondieron a especies de la subfamilia Sigmodontinae: Abrothrix olivaceus, Abrothrix longipilis; Chelemys macronix (Thomas, 1894), Loxodontomys micropus y Oligoryzomys longicaudatus, y dos especies de la sufamilia Murinae: Rattus norvegicus (Berkenhout, 1769) y Rattus rattus (Linnaeus, 1758).

Colcura y Barrio Universitario (ambos de la provincia de Concepción) fueron los sitios con mayor riqueza de especies capturadas, ambos con seis especies. La especie más abundante en términos absolutos fue A. olivaceus $(\mathrm{n}=120)$, seguida de $O$. longicaudatus $(\mathrm{n}=83), A$. longipilis $(\mathrm{n}=46)$ y por el múrido $R$. rattus $(\mathrm{n}=37)$. Las especies con los valores más bajos de abundancia total fueron L. micropus $(\mathrm{n}=22)$, 


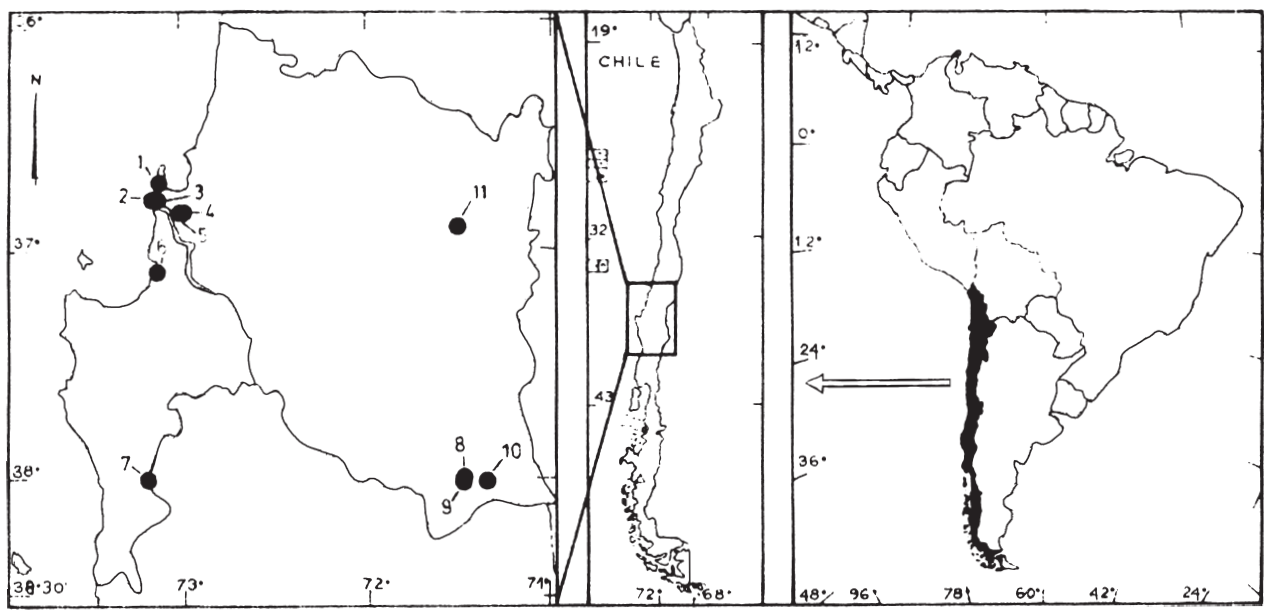

Fig. 1: Localización de los sitios de muestreo: (1) Parque Tumbes; (2) Parque Botánico Hualpén; (3) Parque Pedro del Río Zañartu; (4) Barrio Universitario; (5) Villuco; (6) Colcura; (7) Contulmo; (8) Peralillo (Quilleco); (9) Quepuca-Ralco; (10) Palmucho; (11) Las Trancas.

Location of sampled sites : (1) Parque Tumbes; (2) Parque Botánico Hualpén; (3) Parque Pedro del Río Zañartu; (4) Barrio Universitario; (5) Villuco; (6) Colcura; (7) Contulmo; (8) Peralillo (Quilleco); (9) Quepuca-Ralco; (10) Palmucho; (11) Las Trancas.

TABLA 1

Nombre y ubicación geográfica de las localidades de muestreo, esfuerzo de captura, y densidad relativa* de las especies de roedores capturadas

Name and geographic location of sampled sites, sampling effort, and relative density* of rodent species captured

\begin{tabular}{|c|c|c|c|c|c|c|c|c|c|}
\hline \multirow[t]{2}{*}{ Provincia } & \multirow[t]{2}{*}{ Ubicación geográfica } & \multirow[t]{2}{*}{ Trampas noche } & \multicolumn{7}{|c|}{ Abundancia relativa de las especies capturadas } \\
\hline & & & A lon & A oli & Ch mac & $L \mathrm{mic}$ & O lon & $R$ nor & $R$ rat \\
\hline Arauco & $\begin{array}{l}\text { Contulmo } \\
\left(38^{\circ} 01^{\prime} \mathrm{S}, 73^{\circ} 13^{\prime} \mathrm{O}\right)\end{array}$ & 1.400 & 0,36 & 0,14 & 0,0 & 0,0 & 0,57 & 0,64 & 0,07 \\
\hline \multirow[t]{3}{*}{ Biobío } & $\begin{array}{l}\text { Palmucho } \\
\left(38^{\circ} 03^{\prime} \mathrm{S}, 71^{\circ} 21^{\prime} \mathrm{O}\right)\end{array}$ & 240 & 0,0 & 0,0 & 0,0 & 0,42 & 0,42 & 0,0 & 0,42 \\
\hline & $\begin{array}{l}\text { Quepuca-Ralco } \\
\left(38^{\circ} 01^{\prime} \mathrm{S}, 71^{\circ} 28^{\prime} \mathrm{O}\right)\end{array}$ & 240 & 0,0 & 4,58 & 0,0 & 0,0 & 2,08 & 0,42 & 0,42 \\
\hline & $\begin{array}{l}\text { Peralillo (Quilleco) } \\
\left(37^{\circ} 59^{\prime} \mathrm{S}, 71^{\circ} 28^{\prime} \mathrm{O}\right)\end{array}$ & 320 & 0,0 & 5,31 & 0,0 & 0,0 & 1,25 & 0,31 & 2,5 \\
\hline \multirow[t]{6}{*}{ Concepción } & $\begin{array}{l}\text { Colcura } \\
\left(37^{\circ} 06^{\prime} \mathrm{S}, 73^{\circ} 09^{\prime} \mathrm{O}\right)\end{array}$ & 800 & 0,75 & 3,00 & 0,0 & 0,13 & 1,88 & 0,50 & 0,75 \\
\hline & $\begin{array}{l}\text { Villuco } \\
\left(36^{\circ} 52^{\prime} \mathrm{S}, 73^{\circ} 01^{\prime} \mathrm{O}\right)\end{array}$ & 400 & 1,50 & 7,50 & 0,0 & 1,25 & 6,50 & 0,0 & 0,25 \\
\hline & $\begin{array}{l}\text { Barrio Universitario } \\
\left(36^{\circ} 50^{\prime} \mathrm{S}, 73^{\circ} 03^{\prime} \mathrm{O}\right)\end{array}$ & 320 & 0,31 & 0,94 & 0,0 & 1,25 & 0,63 & 0,31 & 0,94 \\
\hline & $\begin{array}{l}\text { Parque Tumbes } \\
\left(36^{\circ} 42^{\prime} \mathrm{S}, 73^{\circ} 07^{\prime} \mathrm{O}\right)\end{array}$ & 400 & $0, .0$ & 0,0 & 0,0 & 0,0 & 0,75 & 0,25 & 1,25 \\
\hline & $\begin{array}{l}\text { Parque Pedro del Río Zañartu } \\
\left(36^{\circ} 47^{\prime} \mathrm{S}, 73^{\circ} 09^{\prime} \mathrm{O}\right)\end{array}$ & 400 & 2,25 & 3,0 & 0,0 & 0,0 & 3,25 & 0,0 & 2,50 \\
\hline & $\begin{array}{l}\text { Parque Botánico Hualpén } \\
\left(36^{\circ} 47^{\prime} \mathrm{S}, 73^{\circ} 07^{\prime} \mathrm{O}\right)\end{array}$ & 160 & 0,63 & 2,5 & 0,0 & 0,0 & 1,25 & 0,0 & 0,63 \\
\hline Ñuble & $\begin{array}{l}\text { Las Trancas } \\
\left(36^{\circ} 54^{\prime} \mathrm{S}, 71^{\circ} 29^{\prime} \mathrm{O}\right)\end{array}$ & 1000 & 1,7 & 1,7 & 0,2 & 1,1 & 0,4 & 0,0 & 0,0 \\
\hline
\end{tabular}

A lon: Abrothrix longipilis, A oli: Abrothrix olivaceus; Ch mac: Chelemys macronyx; L mic: Loxodontomys micropus; $O$ lon: Oligoryzomys longicaudatus $R$ nor: Rattus norvegicus; $R$ rat: Rattus rattus;.

* (número de capturas de la especie/número total de trampas) x 100 
$R$. norvegicus $(\mathrm{n}=14)$ y Ch. macronyx $(\mathrm{n}=2)$. Los valores de densidad relativa de roedores fluctuaron entre 7,50 y 0,07 , y donde la localidad de Villuco presentó la mayor densidad para A. olivaceus. El valor más bajo se registró en Contulmo para la especie $R$. rattus. Entre los sigmodontinos, L. micropus $(0,13)$ fue el menos abundante en la localidad de Colcura. Las densidades relativas de $O$. longicaudatus (83 individuos capturados) variaron entre 6,5 (Villuco) y 0.4 (Las Trancas; Tabla 1), y fue la única especie presente en los 11 sitios muestreados.

Trescientos de los 326 ejemplares capturados (92\%) fueron analizados serológicamente. El resto de los ejemplares $(n=26)$ no fueron analizados debido a que no fue posible extraer muestras de sangre. Cinco individuos pertenecientes a tres especies fueron positivas a Hantavirus. Estas correspondieron a $O$. longicaudatus (dos individuos, un macho y una hembra adultos) capturados cada uno en las localidades de Quepuca-Ralco y Peralillo; dos individuos (machos adultos) de A. longipilis capturados en Las Trancas; y un individuo (hembra adulta) de $L$. micropus encontrado en Palmucho. Este último dato corresponde al único registro de seropositividad a Hantavirus para esta especie en Chile.

\section{DISCUSIÓN}

El valor de seropositividad a Hantavirus registrado en este estudio alcanzó a un 1,66 \%, una cifra relativamente baja si se compara con valores registrados en otros lugares de Sudamérica, los que han fluctuado entre $3 \%$ para Argentina (Calderón et al. 1999, Cantoni et al. 2001) y $4 \%$ para el chaco Paraguayo (Yahnke et al. 2001). Sin embargo, si se considera solo a los sigmodontinos, nuestros registros son cercanos a 1,97 $\%$, un valor similar a aquellos registrados por otros estudios en Chile (Toro et al. 1998, Pavletic 2000), pero aún menor comparado con registros en Argentina (Calderón et al. 1999, Cantoni et al. 2001). Es posible que esto último esté relacionado a la mayor diversidad de roedores simodontinos en este último país (Redford \& Eisenberg 1992), los que albergarían un mayor número de cepas de Hantavirus (Padula et al. 2000, Bohlman et al. 2002).

A pesar que A. olivaceus fue la especie más abundante en este estudio, no se detectaron individuos seropositivos a Hantavirus, un hecho que es concordante con estudios previos donde la relación seropositividad / abundancia total en esta especie es la más baja entre los roedores señalados como seropositivos (Pavletic 2000). A nivel intraespecífico, tanto L. micropus $(5,26$
$\%$ ) como A. longipilis (4,55 \%) mostraron mayores tasas de seropositividad comparado con O. longicaudatus $(2,5 \%)$. Estos valores son similares al 3,3\% registrado para Oligoryzomys en el norte de Argentina (Pini et al. 2003), aunque bajos si se comparan con un $10 \%$ registrado para Chile (Torres-Pérez et al. 2004) y con un $8 \%$ registrado para el colilargo en Argentina (Calderón et al. 1999).

La proporción sexo / seropositividad ha sido escasamente evaluada, aunque se ha registrado una mayor incidencia de seropositivos en machos (Cantoni et al. 2001), lo que contrasta con los resultados de este estudio donde tres de los cinco ejemplares positivos fueron machos. Es indudable que un mayor esfuerzo temporal de muestreo ayudará a resolver si las diferencias entre sexos detectadas hasta ahora tienen sentido biológico real.

El ejemplar de L. micropus seropositivo a Hantavirus representa el primer registro de este tipo para esta especie en Chile; un segundo ejemplar seropositivo de esta especie se registró en la zona de El Bolsón en Argentina (Cantoni et al. 2001). La baja frecuencia de seropositividad detectada en A. olivaceus podría deberse a una baja tasa de contactos entre esta especie y la especie reservorio, lo que disminuiría la probabilidad de transmisión horizontal del virus mediante "spill-over" (Hjelle \& Yates 2001).

Desde un punto de vista geográfico, cuatro de las 11 localidades muestreadas se encuentran en la precordillera de los Andes (Las Trancas, Palmucho, Quepuca-Ralco y Peralillo), mientras que las restantes, en sectores de la Cordillera de la Costa. La depresión intermedia permanece aún escasamente evaluada. Es precisamente en las localidades andinas (provincias de Ñuble y Biobío) donde se capturaron los cinco ejemplares seropositivos. Estudios posteriores han mostrado roedores positivos a la cepa Andes en sectores andinos precordilleranos de la Octava Región (Torres-Pérez et al. 2004), lo que debiera inducir a un reforzamiento de medidas de prevención sanitarias en estas áreas. Dicho reforzamiento probablemente es más relevante durante el período estival, cuando el número de visitantes en sectores atractivos para el turismo incrementa (Sotomayor \& Aguilera 2000). En base a los mayores valores de seropositividad detectados en la precordillera, sugerimos que los futuros estudios sobre virus Hanta sean dirigidos hacia estas áreas. Un objetivo relevante para estos estudios debiera ser la evaluación de una posible relación entre seropositividad y distribución espacial (e.g., uso de hábitat) y temporal (e.g., estacionalidad) de los roedores. 


\section{AGRADECIMIENTOS}

Este trabajo fue financiado por el Servicio de Salud Nuble y la Dirección de Investigación Universidad de Concepción (DIUC 98.113.463). Debemos agradecer a los Drs. J. Gosa y J.L. Mena (Servicio de Salud de Nuble), D. Osorio (Servicio de Salud de Concepción), S. Briones (Servicio de Salud de Talcahuano), C. Báez (Servicio de Salud de Arauco) y a G. Valladares (Servicio de Salud de Biobío) por su valiosa cooperación en terreno. A la Dra. O. Reyes (Seremi de Salud VIII Región) por su constante apoyo para el buen desarrollo de este proyecto. A la empresa Pangue S.A., al Servicio de Bienestar del Servicio de Salud de Nuble y al Servicio de Salud de Biobío por el apoyo logístico.

\section{LITERATURA CITADA}

BARÓ M, J VERGARA \& M NAVARRETE (1999) Hantavirus en Chile: revisión y análisis de casos desde 1975. Revista Médica de Chile 127: 1513 1523.

BOHLMAN MC, SP MORZUNOV, J MEISSNER M, BETH-TAYLOR, K ISHIBASHI, J ROWE, S LEVIS, D ENRIA, SC ST. JEOR (2002) Analysis of Hantavirus genetic diversity in Argentina: S segment- derived phylogeny. Journal of Virology 76: 3765-3773.

CANTONI G, P PADULA, G CALDERÓN, J MILLS, E HERRERO, P SANDOVAL, V MARTÍNEZ, N PINI \& E LARRIEU (2001) Seasonal variation in prevalence of antibody to to hantaviruses in rodents from southern Argentina. Tropical Medicine and International Health 6: 811-846.

CALDERÓN G, N PINI, J BOLPE, S LEVIS, J MILLS, E SEGURA, N GUTHMANN, G CANTONI, J BECKER, A FONOLLAT, C RIPOLL, M BORTMAN, R BENEDETTI, M SABATTINI \& D ENRIA (1999) Hantavirus reservoir host associated with peridomestic habitats in Argentina. Emerging Infectious Diseases 5: 792-797.

ELGH F, A LUNDKVIST, OA ALEXEYEV, H STENLUND, T AVSIC-ZUPANC, B HJELLE, HW LEE, KJ SMITH, R VAINIONPAA, D WIGER, G WADELL \& P JUTO (1997) Serological diagnosis of Hantavirus infections by enzyme -linked immunosorbent assay based on detection of immunoglobulin $\mathrm{G}$ and $\mathrm{M}$ responses to recombinant nucleocapsid proteins of five viral serotypes. Journal of Clinical Microbiology 35: 1122-1130.

GALLARDO M \& RE PALMA (1990) Systematics of Oryzomys longicaudatus (Rodentia, Muridae) in Chile. Journal of Mammalogy 71: 333-342.

GONZALEZ LA, R MURÚA \& C JOFRÉ (2000) Habitat utilization of two muroid species in relation to population outbreaks in southern temperate forests of Chile. Revista Chilena de Historia Natural 73: 489-495.

GONZÁLES-ITTIG R, G THEILER \& C GARDENAL (2002) A contribution to the subgeneric systematics of Oligoryzomys (Rodentia, Muridae) from Argentina by means of PCR-RFLP patterns of mitochondrial DNA. Biochemical Systematics and Ecology 30: 23-33.
HJELLE B \&T YATES (2001) Modeling Hantavirus maintenance and transmission in rodent communities. Current Topics in Microbiology and Immunology 256: 77-90.

LEVIS S, S MORZUNOV, J ROWE, D ENRIA, N PINI, G CALDERÓN, M SABATTINI \& SC ST. JEOR (1998) Genetic diversity and epidemiology of hantaviruses in Argentina. Journal of Infectious Diseases 177: 529-538

MANN G (1978) Los pequeños mamíferos de Chile. Gayana Zoología (Chile) 40: 1-342.

MILLS J \& J CHILDS (1998) Ecological studies of rodents reservoirs: their relevance for human health. Emerging Infectious Diseases 4: 529-537.

MILLS J, J CHILDS, T KSIAZEK, CJ PETERS \& WM VELLECA (1998) Métodos para trampeo y muestreo de pequeños mamíferos para estudio virológico. Organización Panamericana de la Salud, Washington, District of Columbia, USA. Report \#OPS/HPS/HCT98.104.

MURÚA R, LA GONZÁLEZ \& PL MESERVE (1987) Populations ecology of Oryzomys longicaudatus philiphii (Rodentia: Cricetidae) in southern Chile. Journal of Animal Ecology 55: 281-293.

OSGOOD W (1943) The mammals of Chile. Publication of the Field Museum of Natural History, Zoological Series 30: 1-268.

PADULA PJ, SB COLAVECCHIA, VP MARTÍNEZ, MO GONZALES, A ELDELSTEIN, DL MIGUEL, J RUSSI, J MORA-RIQUELME, N COLUCCI, M ALMIRÓN \& RD RABINOVICH (2000) Genetic diversity, distribution, and serological features of hantavirus infection in five countries in South America. Journal of Clinical Microbiology 38: 3029-3035.

PAVLETIC C (2000) Hantavirus: su distribución geográfica entre los roedores silvestres de Chile. Revista Chilena de Infectología 17: 186-196.

PINI N, S LEVIS, G CALDERÓN, J RAMÍREZ, D BRAVO, E LOZANO, C RIPOLL, S ST. JEOR, TG KSIAZEK, RM BARQUEZ \& D ENRIA (2003) Hantavirus infection in humans and rodents, northwestern Argentina. Emerging Infectious Diseases 9: 1070-1076.

REDFORD KH \& JF EISENBERG (1992) Mammals of the Neotropics. The Southern Cone. The University Chicago Press, Chicago, Illinois, USA. 430 pp.

SCHMALJOHN C \& B HJELLE (1997) Hantaviruses: a global disease problem. Emerging Infectious Diseases 3: 95-104.

SOTOMAYOR V \& X AGUILERA (2000) Epidemiología de la infección humana por Hantavirus en Chile. Revista Chilena de Infectología 17: 220-232.

SPORTORNO A, RE PALMA \& J VALLADARES (2000) Biología de roedores reservorios de Hantavirus en Chile. Revista Chilena de Infectología 17: 197-210.

TORO J, JD VEGA, AS KHAN, JN MILLS, P PADULA, W TERRY, Z YADÓN, R VALDERRAMA, BA ELLIS, C PAVLETIC, R CERDA, S ZAKI, S WUN-JU, R MEYER, M TAPIA, C MANSILLA, M BARÓ, JA VERGARA, M CONCHA, G CALDERÓN, D ENRIA, CJ PETERS \& TG KSIAZEK (1998) An outbreak of Hantavirus Pulmonary Syndrome, Chile, 1997. Emerging Infectious Diseases 4: 687-694.

TORRES-PÉREZ F, J NAVARRETE-DROGUETT, R ALDUNATE, TL YATES, GJ MERTZ, PA VIAL, M FERRÉS, PA MARQUET \& RE PALMA (2004) Peridomestic small mammals associated with confirmed cases of human Hantavirus disease in southcentral Chile. American Journal of Tropical Medicine and Hygiene 70: 305-309. 
YATES TL, JN MILLS, CA PARMENTER, TG KSIAZEK, RR PARMENTER, JR VANDE CASTLE, CH CALISHER, ST NICHOL, KD ABBOT, JC YOUNG, ML MORRISON, BJ BAETY, JL DUNNUM, RJ BAKER, J SALAZARBRAVO \& CJ PETERS (2002) The ecology and evolutionary history of an emergent disease:
Hantavirus Pulmonary Syndrome. BioScience 52: 989-998

YAHNKE CJ, PL MESERVE, TG KSIAZEK \& JN MILLS (2001) Patterns of infection with Laguna Negra virus in wild populations of Calomys laucha in the central Paraguayan chaco. American Journal of Tropical Medicine and Hygiene 65: 768-776. 